International Journal of Pure and Applied Mathematics

Volume 85 No. 3 2013, 487-494

ISSN: 1311-8080 (printed version); ISSN: 1314-3395 (on-line version)

url: http://www.ijpam.eu

doi: http://dx.doi.org/10.12732/ijpam.v85i3.5

ijpam.eu

\title{
NEW IDENTITIES FOR THE COMMON FACTORS OF BALANCING AND LUCAS-BALANCING NUMBERS
}

\author{
Prasanta Kumar Ray \\ International Institute of Information Technology \\ Gothapatna, PO: MALIPADA, Bhubaneswar,751 003, INDIA
}

\begin{abstract}
Balancing numbers $n$ and balancers $r$ are originally defined as the solution of the Diophantine equation $1+2+\cdots+(n-1)=(n+1)+(n+$ $2)+\cdots+(n+r)$. If $n$ is a balancing number, then $8 n^{2}+1$ is a perfect square. Further, If $n$ is a balancing number then the positive square root of $8 n^{2}+1$ is called a Lucas-balancing number. These numbers can be generated by the linear recurrences $B_{n+1}=6 B_{n}-B_{n-1}$ and $C_{n+1}=6 C_{n}-C_{n-1}$ where $B_{n}$ and $C_{n}$ are respectively denoted by the $n^{\text {th }}$ balancing number and $n^{\text {th }}$ Lucas-balancing number. In this study, we establish some new identities for the common factors of both balancing and Lucas-balancing numbers.
\end{abstract}

AMS Subject Classification: 11B39, 11B83

Key Words: balancing numbers, Lucas-balancing numbers, recurrence relation

\section{Introduction}

Behera and Panda [1] recently introduced a number sequence called balancing numbers defined in the following way: A positive integer $n$ is called a balancing number with balancer $r$, if it is the solution of the Diophantine equation $1+$ $2+\ldots+(n-1)=(n+1)+(n+2)+\ldots+(n+r)$. They also proved that the

Received: January 11, 2013

(c) 2013 Academic Publications, Ltd. url: www.acadpubl.eu 
recurrence relation for balancing numbers is

$$
B_{n+1}=6 B_{n}-B_{n-1}, \quad n>2,
$$

where $B_{n}$ is the $n^{\text {th }}$ balancing number with $B_{1}=1$ and $B_{2}=6$.

It is well known that (see [1]), $n$ is a balancing number if and only if $n^{2}$ is a triangular number, that is $8 n^{2}+1$ is a perfect square. In [10], Lucas-balancing numbers are defined as follows: If $n$ is a balancing number, $C_{n}=\sqrt{8 n^{2}+1}$ is called a Lucas-balancing number. The recurrence relation for Lucas-balancing numbers is same as that of balancing numbers, that is

$$
C_{n+1}=6 C_{n}-C_{n-1}, \quad n>2,
$$

where $C_{n}$ is the $n^{\text {th }}$ Lucas-balancing number with $C_{1}=3$ and $C_{2}=17$. Liptai [4], showed that the only balancing number in the sequence of Fibonacci numbers is 1. In [11] and [12], Ray obtain nice product formulas for both balancing and Lucas-balancing numbers. Panda and Ray [8], link balancing numbers with Pell and associated Pell numbers. They shown that balancing numbers are indeed the product of Pell and associated Pell numbers. Many interesting properties and important identities are available in the literature. Interested readers can follow $[2,3,5,6,7,13,14]$.

The closed form of both balancing and Lucas-balancing numbers are respectively given by

$$
B_{n}=\frac{\lambda_{1}^{n}-\lambda_{2}^{n}}{\lambda_{1}-\lambda_{2}}
$$

and

$$
C_{n}=\frac{\lambda_{1}^{n}+\lambda_{2}^{n}}{2}
$$

for $n \geq 1$ with $\lambda_{1}=3+\sqrt{8}, \lambda_{2}=3-\sqrt{8}$. These relations (1.3) and (1.4) are popularly known as Binet's formulas for balancing and Lucas-balancing numbers. In this paper, we obtain some new identities for the common factors of these numbers.

\section{New Identities for the Common Factors of Balancing and Lucas-Balancing Numbers}

In this section, we present some new identities for the common factors of both balancing and Lucas-balancing numbers with the help of Binet's formula. It is clear that

$$
\lambda_{1}+\lambda_{2}=6, \quad \lambda_{1}-\lambda_{2}=2 \sqrt{8}, \quad \lambda_{1} \lambda_{2}=1 .
$$


Theorem 2.1. For $n \geq 1$, the following identity is valid:

$$
B_{4 n}-6=2 B_{2 n-1} C_{2 n+1} .
$$

Proof. By (2.1), we obtain

$$
\begin{aligned}
2 B_{2 n-1} C_{2 n+1} & =2 \frac{\lambda_{1}^{2 n-1}-\lambda_{2}^{2 n-1}}{\lambda_{1}-\lambda_{2}} \frac{\lambda_{1}^{2 n+1}+\lambda_{2}^{2 n+1}}{2} \\
& =\frac{\lambda_{1}^{4 n}-\lambda_{2}^{4 n}}{\lambda_{1}-\lambda_{2}}-\frac{\lambda_{1}^{2}-\lambda_{2}^{2}}{\lambda_{1}-\lambda_{2}} \\
& =B_{4 n}-6
\end{aligned}
$$

which finishes the proof.

Theorem 2.2. For $n \geq 1$, the following identity is valid:

$$
B_{4 n+1}+1=2 B_{2 n+1} C_{2 n} .
$$

Proof. By (2.1), we get

$$
\begin{aligned}
2 B_{2 n+1} C_{2 n} & =2 \frac{\lambda_{1}^{2 n+1}-\lambda_{2}^{2 n+1}}{\lambda_{1}-\lambda_{2}} \frac{\lambda_{1}^{2 n}+\lambda_{2}^{2 n}}{2} \\
& =\frac{\lambda_{1}^{4 n+1}-\lambda_{2}^{4 n+1}}{\lambda_{1}-\lambda_{2}}+\frac{\left(\lambda_{1} \lambda_{2}\right)^{n} \lambda_{1}-\left(\lambda_{1} \lambda_{2}\right)^{n} \lambda_{2}}{\lambda_{1}-\lambda_{2}} \\
& =B_{4 n+1}+1
\end{aligned}
$$

which is the end of the proof.

Theorem 2.3. For $n \geq 1$, the following identity is valid:

$$
B_{4 n+2}+6=2 B_{2 n+2} C_{2 n} .
$$

Proof. By (2.1), we have

$$
\begin{aligned}
2 B_{2 n+2} C_{2 n} & =2 \frac{\lambda_{1}^{2 n+2}-\lambda_{2}^{2 n+2}}{\lambda_{1}-\lambda_{2}} \frac{\lambda_{1}^{2 n}+\lambda_{2}^{2 n}}{2} \\
& =\frac{\lambda_{1}^{4 n+2}-\lambda_{2}^{4 n+2}}{\lambda_{1}-\lambda_{2}}+\left(\lambda_{1} \lambda_{2}\right)^{2 n} \frac{\lambda_{1}^{2}-\lambda_{2}^{2}}{\lambda_{1}-\lambda_{2}} \\
& =B_{4 n+2}+6
\end{aligned}
$$

which is the end of the proof. 
By the same way, we have the following result.

Theorem 2.4. For $n \geq 1$, the following identity is valid:

$$
B_{4 n+3}-1=2 B_{2 n+1} C_{2 n+2} .
$$

The following lemma is already established in[8]. For the sake of simplicity we present the proof again.

Lemma 2.5. For $n \geq 1$, the following identity is valid:

$$
B_{2 n}=2 B_{n} C_{n}
$$

Proof. By (2.1), we get

$$
\begin{aligned}
2 B_{n} C_{n} & =2 \frac{\lambda_{1}^{n}-\lambda_{2}^{n}}{\lambda_{1}-\lambda_{2}} \frac{\lambda_{1}^{n}+\lambda_{2}^{n}}{2} \\
& =\frac{\lambda_{1}^{2 n}-\lambda_{2}^{2 n}}{\lambda_{1}-\lambda_{2}} \\
& =B_{2 n}
\end{aligned}
$$

which completes the proof.

Lemma 2.6. For $n \geq 1$, the following identity is valid:

$$
B_{4 n+1}-1=2 B_{2 n} C_{2 n+1} \text {. }
$$

Proof. By (2.1), we have

$$
\begin{aligned}
2 B_{2 n} C_{2 n+1} & =2 \frac{\lambda_{1}^{2 n}-\lambda_{2}^{2 n}}{\lambda_{1}-\lambda_{2}} \frac{\lambda_{1}^{2 n+1}+\lambda_{2}^{2 n+1}}{2} \\
& =\frac{\lambda_{1}^{4 n+1}-\lambda_{2}^{4 n+1}}{\lambda_{1}-\lambda_{2}}-\left(\lambda_{1} \lambda_{2}\right)^{2 n} \frac{\lambda_{1}-\lambda_{2}}{\lambda_{1}-\lambda_{2}} \\
& =B_{4 n+1}-1
\end{aligned}
$$

which is the end of the proof.

By virtue of Lemma 2.5 and Lemma 2.6, we have the following result.

Corollary 2.7. For $n \geq 1$, we have

$$
B_{4 n+1}-1=2 B_{n} C_{n} C_{2 n+1} .
$$


Lemma 2.8. For $n \geq 1$, the following identity is valid:

$$
C_{4 n+1}-3=16 B_{2 n} B_{2 n+1} .
$$

Proof. Since $\left(\lambda_{1}-\lambda_{2}\right)^{2}=32$, we get

$$
\begin{aligned}
16 B_{2 n} B_{2 n+1} & =16 \frac{\lambda_{1}^{2 n}-\lambda_{2}^{2 n}}{\lambda_{1}-\lambda_{2}} \frac{\lambda_{1}^{2 n+1}-\lambda_{2}^{2 n+1}}{\lambda_{1}-\lambda_{2}} \\
& =\frac{\lambda_{1}^{4 n+1}+\lambda_{2}^{4 n+1}}{2}-\left(\lambda_{1} \lambda_{2}\right)^{2 n} \frac{\lambda_{1}+\lambda_{2}}{2} \\
& =C_{4 n+1}-3
\end{aligned}
$$

which ends the proof.

Since $B_{2 n}=2 B_{n} C_{n}$, the following identity is valid for $n \geq 1$ :

Theorem 2.9.

$$
C_{4 n+1}-3=2 C_{2 n} C_{2 n+1} .
$$

Theorem 2.10. For $n \geq 1$, the following identity is valid:

$$
C_{4 n+1}+3=2 C_{2 n} C_{2 n+1} .
$$

Proof. By (2.1), we have

$$
\begin{aligned}
2 C_{2 n} C_{2 n+1} & =2 \frac{\lambda_{1}^{2 n}+\lambda_{2}^{2 n}}{2} \frac{\lambda_{1}^{2 n+1}+\lambda_{2}^{2 n+1}}{2} \\
& =\frac{\lambda_{1}^{4 n+1}+\lambda_{2}^{4 n+1}}{2}+\left(\lambda_{1} \lambda_{2}\right)^{2 n} \frac{\lambda_{1}+\lambda_{2}}{2} \\
& =C_{4 n+1}+3
\end{aligned}
$$

which is the end of the proof.

Lemma 2.11. For $n \geq 1$, the following identity is valid:

$$
B_{4 n+3}+1=2 B_{2 n+2} C_{2 n+1} .
$$

Proof. By using (2.1), we have

$$
\begin{aligned}
2 B_{2 n+2} C_{2 n+1} & =2 \frac{\lambda_{1}^{2 n+2}-\lambda_{2}^{2 n+2}}{\lambda_{1}-\lambda_{2}} \frac{\lambda_{1}^{2 n+1}+\lambda_{2}^{2 n+1}}{2} \\
& =\frac{\lambda_{1}^{4 n+2}-\lambda_{2}^{4 n+2}}{\lambda_{1}-\lambda_{2}}+\left(\lambda_{1} \lambda_{2}\right)^{2 n+1} \\
& =B_{4 n+3}+1
\end{aligned}
$$

which completes the proof. 
Theorem 2.12. For $n \geq 1$, we have

$$
B_{4 n+3}+1=4 B_{n+1} C_{n+1} C_{2 n+1} .
$$

Proof. Substituting $B_{2 n+2}=2 B_{n+1} C_{n+1}$ from Lemma 2.5 into Lemma 2.11, we obtain the desired result.

Theorem 2.13. For $n \geq 1$, the following identity is valid:

$$
C_{4 n+3}+3=2 C_{2 n+1} C_{2 n+2} \text {. }
$$

Proof. By (2.1), we have

$$
\begin{aligned}
2 C_{2 n+1} C_{2 n+2} & =2 \frac{\lambda_{1}^{2 n+1}+\lambda_{2}^{2 n+1}}{2} \frac{\lambda_{1}^{2 n+2}+\lambda_{2}^{2 n+2}}{2} \\
& =\frac{\lambda_{1}^{4 n+3}+\lambda_{2}^{4 n+3}}{2}+\left(\lambda_{1} \lambda_{2}\right)^{2 n+1} \frac{\lambda_{1}+\lambda_{2}}{2} \\
& =C_{4 n+3}+3
\end{aligned}
$$

which completes the proof.

Theorem 2.14. For $n \geq 1$, the following identity is valid:

$$
C_{4 n+3}-3=16 B_{2 n+1} B_{2 n+2} \text {. }
$$

Proof. Since $\left(\lambda_{1}-\lambda_{2}\right)^{2}=32$, we obtain

$$
\begin{aligned}
16 B_{2 n+1} B_{2 n+2} & =2 \frac{\lambda_{1}^{2 n+1}-\lambda_{2}^{2 n+1}}{\left.\lambda_{1}-\lambda_{2}\right)} \frac{\lambda_{1}^{2 n+2}-\lambda_{2}^{2 n+2}}{\lambda_{1}-\lambda_{2}} \\
& =\frac{\lambda_{1}^{4 n+3}+\lambda_{2}^{4 n+3}}{2}-\left(\lambda_{1} \lambda_{2}\right)^{2 n+1} \frac{\lambda_{1}+\lambda_{2}}{2} \\
& =C_{4 n+3}-3
\end{aligned}
$$

which ends the proof.

The following corollary is an immediate consequence of Theorem 2.14.

Corollary 2.15. For $n \geq 1$, we have

$$
C_{4 n+3}-3=32 B_{n+1} C_{n+1} B_{2 n+1} .
$$




\section{References}

[1] A. Behera and G.K. Panda, On the square roots of triangular numbers, The Fibonacci Quarterly, 37, No. 2 (1999), 98-105.

[2] A. Berczes, K. Liptai, I. Pink, On generalized balancing numbers, Fibonacci Quarterly, 48, No. 2 (2010), 121-128.

[3] R. Keskin, O. Karaatly, Some new properties of balancing numbers and square triangular numbers, Journal of Integer Sequences, 15, No. 1 (2012).

[4] K. Liptai, Fibonacci balancing numbers, The Fibonacci Quarterly, 42, No. 4 (2004), 330-340.

[5] K. Liptai, Lucas balancing numbers, Acta Math.Univ. Ostrav, 14, No. 1 (2006), 43-47.

[6] K. Liptai, F. Luca, A. Pinter, L. Szalay, Generalized balancing numbers, Indagationes Math. N. S., 20 (2009), 87-100.

[7] P. Olajos, Properties of balancing, cobalancing and generalized balancing numbers, Annales Mathematicae et Informaticae, 37 (2010), 125-138.

[8] G.K. Panda, P.K. Ray, Some links of balancing and cobalancing numbers with Pell and associated Pell numbers, Bulletin of the Institute of Mathematics, Academia Sinica (New Series), 6, No. 1 (2011), 41-72.

[9] G.K. Panda, P.K. Ray, Cobalancing numbers and cobalancers, International Journal of Mathematics and Mathematical Sciences, 8 (2005), 11891200 .

[10] G.K. Panda, Some fascinating properties of balancing numbers, Proc. Eleventh Internat. Conference on Fibonacci Numbers and Their Applications, Cong. Numerantium, 194 (2009), 185-189.

[11] P.K. Ray, Application of Chybeshev polynomials in factorization of balancing and Lucas-balancing numbers, Bol. Soc. Paran. Mat., 30, No. 2 (2012), 49-56.

[12] P.K. Ray, Factorization of negatively subscripted balancing and Lucasbalancing numbers, Bol.Soc.Paran.Mat., 31, No. 2 (2013), 161-173.

[13] P.K. Ray, Curious congruences for balancing numbers, Int. J. Contemp. Sciences, 7, No. 18 (2012), 881-889. 
[14] P.K. Ray, Certain matrices associated with balancing and Lucas-balancing numbers, Matematika, 28, No. 1 (2012), 15-22. 